\title{
Randomized Authentication Systems
}

\author{
Josef Pieprzyk \\ Reihaneh Safavi-Naini*
}

Department of Computer Science

University College

University of New South Wales

Australian Defence Force Academy

Canberra, ACT 2600, AUSTRALIA

\author{
Department of Mathematic, Statistic and Computing \\ University of New England \\ Armidale, NSW 2351, AUSTRALIA
}

\begin{abstract}
In this work, the application of Luby-Rackoff randomizers for authentication purposes is examined. First randomized authentication codes are introduced. In these codes, the assignment of a cryptogram to a given message is done in two stages. In the first, the redundancy is introduced and in the second, the concatenation of several Luby-Rackoff randomizers is used. Next, perfect $A$-codes are defined. The quality of the authentication codes ( $A$-codes) is measured using the concept of distinguishing circuits. Three $A$-codes with different redundancy stages are examined and proven that they are perfect if the suitable number of Luby-Rackoff randomizers is used in the second stage of the $A$-code.
\end{abstract}

\section{Introduction}

We are going to consider authentication systems whose quality can be rigorously proven. It is known [8] that randomizers can be applied to construct provably secure cryptosystems. The hain quality measurements of such cryptosystems is their indistinguishability from truly randc $m$ generators ([2], [7]).

We construct a class of authentication systems (codes) that consists of two stages. The first stage introduces redundancy to the message by applying some encoding rule $([3],[4],[5],[6])$ while the second stage spreads this redundancy over the whole cryptogram.

To be more precise, we assume that messages are $n$-bit long and we add the redundancy by concatenating $(m-n)$-bit strings to the message $(m>n)$. The resulting message of length

\footnotetext{
'Support for this project was provided in part by TELECOM Australia under the contract number 7027 and by the Australian Research Council under the reference number A48830241.
} 
$m$ (later we consider the case when $m=2 n$ ) is submitted to the second stage which is a randomizer. The resulting cryptogram of length $m$ is transmitted to the receiver over a publicly exposed channel ([5]).

The receiver first recovers the redundant message and next compares the $(m-n)$-bit redundant string to the original one. They accept the messages as authentic only if the two redundant strings are the same.

\section{Preliminaries}

Let $I_{n}=\{0,1\}^{n}$ be the set of all $2^{n}$ binary strings of length $n$ and let the set $F_{n, m}$ of all functions from $I_{n}$ to $I_{m}$ be defined as

$$
F_{n, m}=\left\{f \mid f: I_{n} \rightarrow I_{m}\right\} .
$$

$F_{n, m}$ consists of $2^{m 2^{n}}$ elements (we will use $F_{n}$ for the case $m=n$ ). In general, $f \in F_{n, m}$ might not have an inverse. In fact if $n>m$ then such an inverse function doesn't exist.

The definition of a generalized function generator can be given similar to that of a function generator (see [1], [2]).

Deflnition 2.1 Let $l(n)$ and $m(n)$ be polynomials in $n$. A generalized function generator with index $k$ of length $l(n)$ is a collection $\mathbf{f}=\left\{f^{n, m}\right\}$ where the index $k$ specifies a function $f_{k}^{n, m} \in$ $f^{n, m}$. For given index $k \in I_{l(n)}$ and $x \in I_{n}$, the function $f_{k}^{n, m}(x)$ is computable in polynomial time.

Also the concept of distinguishing circuit family can be extended to this case:

Definition 2.2 A distinguishing circuit $C_{n, m}$ for $f^{n, m}$ is an acyclic circuit which consists of Boolean gates (AND, OR, NOT), constant gates (" $O$ " and " 1 ") and $r$ oracle gates $\left(r<2^{n}\right)$. The circuit has one bit output only. Oracle gates accept binary inputs of length $n$ and generate binary outputs of the length $m$. Each oracle gate is evaluated using some function from the space $F_{n, m}$.

Deflnition 2.3 A family of distinguishing circuits for $\left\{f^{n, m}\right\}$ is an infinite sequence of circuits $C_{n_{1}, m_{1}}, C_{n_{2}, m_{2}}, \cdots\left(n_{1}<n_{2}<\cdots\right)$ such that for two constants $c_{1}$ and $c_{2}$ and for each pair of parameters $n, m$, there exists a circuit $C_{n, m}$ which has the following properties:

- The size of $C_{n, m}$ is smaller than $n^{c_{1}}$ (the size is defined as the number of all connections between gates).

- Let $\operatorname{Pr}\left[C_{n, m}\left(F_{n, m}\right)\right]$ be the probability that the output bit of $C_{n, m}$ is one when a function is randomly and uniformly selected from $F_{n, m}$ and used to evaluate the oracle gates. Let $\operatorname{Pr}\left[C_{n, m}\left(f^{n, m}\right)\right]$ be the probability that the output bit of $C_{n, m}$ is one when the oracle gates are evaluated using a function randomly and uniformly selected from $f^{n, m}$ (by random selection of the index $k$ ). The robability of distinguishing the truly random function from the one generated by $f^{n, m}$, is

$$
\left|\operatorname{Pr}\left[C_{n, m}\left(F_{n, m}\right)\right]-\operatorname{Pr}\left[C_{n, m}\left(f^{n, m}\right)\right]\right| \geq \frac{1}{n^{c_{2}}}
$$

We say that a function generator $\left\{f^{n, m}\right\}$ does not have a family of distinguishers if for almost all parameters $n$

$$
\left|\operatorname{Pr}\left[C_{n, m}\left(F_{n, m}\right)\right]-\operatorname{Pr}\left[C_{n, m}\left(f^{n, m}\right)\right]\right| \leq \frac{1}{n^{c_{2}}}=\frac{p(r)}{2^{n}}
$$


where $r$ is the number of oracle gates in the distinguisher and $p(r)$ is a polynomial in $r$. A generalized pseudorandom function generator is a generalized function generator that does not have a family of distinguishing circuits.

The construction of a pseudorandom function generator from a cryptographically secure pseudorandom bit generator (CSB) is given in [1] where it is noted that the construction is extendable to generalized pseudorandom function generators.

For a function $f \in F_{n}$, we can determine a DES-type permutation $D_{f} \in F_{2 n}$ as

$$
D_{f}(L, R)=(R, L \oplus f(R))
$$

where $L$ and $R$ are $n$-bit input strings $\left(L, R \in I_{n}\right.$ ). Having a sequence of functions $f_{1}, f_{2}, \cdots, f_{i} \in$ $F_{n}$, we can determine the concatenation of their DES-type permutations $\psi$ and

$$
\psi\left(f_{1}, f_{2}, \cdots, f_{i}\right)=D_{f_{i}} \circ D_{f_{i-1}} \circ \cdots \circ D_{f_{i}}
$$

Of course, $\psi\left(f_{1}, f_{2}, \cdots, f_{i}\right) \in F_{2 n}$.

Note that a generator $\psi\left(f_{1}, \ldots, f_{i}\right)$ (where $f_{j} \in F_{n}$ for all $j=1,2, \ldots, i$ ) should be seen as a class of permutations for different parameters $n=1,2,3, \ldots$

Obviously, the quality of permutation generators $\psi\left(f_{1}, \cdots, f_{i}\right)$ depends upon the selection of their functions $f_{j}(1 \leq j \leq i)$. Luby and Rackoff [2] showed that $\psi(f, g, h)$ does not have a distinguisher and

$$
\left|\operatorname{Pr}\left[C_{n, n}\left(F_{2 n}\right)\right]-\operatorname{Pr}\left[C_{n, n}(\psi(f, g, h))\right]\right| \leq \frac{r^{2}}{2^{n}}
$$

where $r \leq 2^{n}$ is the number of oracle gates in a distinguishing circuit and the functions $f, g, h$ are randomly and uniformly selected from the space $F_{n}$ i.e. $f, g, h \in_{R} F_{n}$. It means that for all possible distinguishing circuits $C_{2 n}$ which contain $r$ oracle gates (all statistical tests with the access to $r$ observations of input/output pairs), the probabilities $\operatorname{Pr}\left[C_{2 n}\left(F_{2 n}\right)\right], \operatorname{Pr}\left[C_{2 n}(\psi(f, g, h))\right]$ are very close to each other and their difference can be made as small as required by selecting a large enough parameter $n$.

The Luby-Rackoff module $\psi(f, g, h)$ can be used in two different ways, as a pseudorandom permutation generator when the three functions $f, g, h$ are pseudorandom (it will be called L-R module), and as a randomizer when $f, g, h$ are randomly and uniformly selected from $F_{n}$ (it will be called L-R randomizer).

Note that a L-R randomizer no longer constitutes a pseudorandom function generator as the three random functions $f, g, h$ create an index whose length is not polynomial in $n$. Therefore for a given index and input $x \in I_{n}$, the output is not computable in polynomial time.

\section{Perfect Authentication Codes}

\section{$3.1 \quad$ A-Codes}

Authentication codes ( $A$-codes) were introduced by Simmons [6] in the study of authentication systems. An $A$-code $A^{n, m}=\left\{A_{k}^{n, m}\right\}$ is a set of invertible one-to-one functions from $I_{n}$ to $I_{m}$, where each function is indexed by a key $k$. It is required that if $k$ is known, $A_{k}^{n, m}(x)\left(x \in I_{n}\right)$ can be computed in polynomial time. An $A$-code can provide security only if $n<m$, i.e., there is redundancy in the system.

There is an attack on authentication systems that is called a spoofing of order $T$. In this attack, an enemy has intercepted $T$ cryptograms and attempts an impersonation or substitution attack on the system. We say that an $A$-code provides security of order $\mathrm{T}$ ( $\mathrm{T}$ a polynomial in $n$ ) if for infinitely many $n$, the best strategy of an enemy in the spoofing of order $T$ attack on 
$\left\{A^{n, m}\right\}$ is random selection with uniform distribution from the set $I_{m}$ when the $T$ intercepted cryptograms are excluded.

So for security against the spoofing of order $T$, it is required that the enemy cannot use their knowledge of the $T$ intercepted cryptograms in designing a better strategy. Using a distinguishing circuit family for assessing $A$-code generators is in fact equivalent to an attack stronger than spoofing, i.e., the attack in which pairs of message/cryptogram are accessible to the enemy. Moreover the distinguishing circuit (the attacker) can choose messages and collect cryptograms from the oracle gates. We assume that the distinguishing circuit computing resources (the attacker's resources) are not polynomially bounded but the number of accessible pairs of message/cryptogram (the number of oracle gates) is limited.

\subsection{Randomized $A$-Codes}

We give the construction of randomized $A$-codes (also called randomized authentication systems). The construction has two stages (see [4]). The first stage introduces redundancy while the second stage serves to diffuse the redundant information over the whole block of cryptogram using the concatenation of several $L-R$ randomizers.

Definition 3.1 $A$ randomized $A$-code $\mathcal{A}_{n}=\left\{A^{n, 2 n}\right\}$ is a family of functions $A^{n, 2 n}=\left\{A_{k}^{n, 2 n}\right\} \subset$ $F_{n, 2 n}$. The essignment of the cryptogram (the output) for a given message (the input) $M \in I_{n}$, is done in the following two stages:

- in the first stage redundancy is introduced into the message $M \in I_{n}$ by creating the redun. dant message $M^{\prime}=(M, \vartheta) \in I_{2 n}$ where $\vartheta$ is a string of redundant bits added to $M$;

- in the second stage, the message $M^{\prime}$ is transformed using the cancatenation of $i$ ( $i=$ $1,2, \ldots) L-R$ randomizers $\psi(f, g, h)\left(f, g, h \in_{R} F_{n}\right)$. The resulting randomizer is $\Psi_{i}=$ $\underbrace{\psi \circ \psi \circ \cdots \circ \psi}$. The random functions $f, g, h$ are a part of the system cryptographic keys $\underbrace{i}_{i}$ and are kept secret (known to the sender and receiver only).

Clearly, randomized $A$-codes are not pseudorandom function generators as they are based upon L-R randomizers for which neither the indexing nor the polynomial time evaluation are satisfied. However, the quality of randomized $A$-codes can be assessed by using distinguishing circuits $\left\{C_{n, m}\right\}$ as given in Definition $2.3(m=2 n)$.

In the following, we define a class of perfect randomized $A$-codes.

Deflnition 3.2 $A$ randomized $A$-code $\mathcal{A}_{n}=\left\{A^{n, 2 n}\right\}$ is perfect if for any distinguisher $C_{n, 2 n}$, all outputs of the oracle gates (evaluated by the A-code) are independent from input random variables.

Let us consider a randomized $A$-code $\mathcal{A}_{n}=\left\{A^{n, 2 n}\right\}$ for which in the first stage for each message $M f \in I_{n}, \vartheta$ is selected independently and with the uniform probability from the space $I_{n}$ (the selected $\vartheta$ is kept secret). In the second stage, there is a single L-R randomizer. The quality of the $A$-code is given by the following theorem.

Theorem $3.1 A$ single $L-R$ randomizer $\psi_{1}\left(f, g^{*}, h\right)$ is enough $\left(\Psi_{1}=\psi_{1}\left(f, g^{*}, h\right), f, h \in_{R} F_{n}\right.$ and $g^{*} \in_{R} F_{n}$ is a random permutation) for the second stage of $\mathcal{A}_{n}=\left\{A^{n, 2 n}\right\}$ to obtain a perfect randomized $A$-code provided that in the first stage for each message $M_{i} \in I_{n}$, there is an independent and uniformly distributed random variable $\vartheta_{i}$ (the distinguisher has $r \leq 2^{n}$ oracle gates). 
Proof. If we have $r\left(r \leq 2^{n}\right)$ oracle gates, then all oracle gates should produce $r$ independent random variables. This happens only if for any pair of oracle gates $\left(O_{i}, O_{j}\right)(0 \leq i<j \leq r)$, their outputs are independent from their inputs. Let us consider a single pair of oracle gates $\left(O_{i}, O_{j}\right)$. They are fed by two different messages $M_{i}, M_{j}$ (they are random variables which can take on different values) and the $A$-code generate two independent random variables $\vartheta_{i}, \vartheta_{j}$ for them (note that $\vartheta_{i}$ and $\vartheta_{j}$ are not accessible to the distinguisher). If random variables $\vartheta_{i}$ and $\vartheta_{j}$ are independent and uniform, then the outputs $y_{i_{1}}$ and $y_{j_{1}}$ are independent as well (see Figure 1). Now consider random variables $y_{i_{2}}$ and $y_{j_{2}}$. We have the two following cases.

(a) If random variables $\vartheta_{i}$ and $\vartheta_{j}$ take on different values. It results that the function $f$ assigns two independent random variables. So $y_{i_{2}}$ and $y_{j_{2}}$ are independent $\left(\vartheta_{i}\right.$ and $\vartheta_{j}$ are not accessible to the distinguisher) from the input random variables $x_{i_{1}}, x_{j_{1}}$.

(b) If $\vartheta_{i}$ and $\vartheta_{j}$ have happened to have the same value $\vartheta=\vartheta_{i}=\vartheta_{j}$. Then $\alpha_{i}=M_{i} \oplus f(\vartheta)$ and $\alpha_{j}=M_{j} \oplus f(\vartheta)$. As random variables $\alpha_{i}, \alpha_{j}$ can take on different values only, the outputs $y_{i_{1}}=g^{*}\left(\alpha_{i}\right) \oplus \vartheta, y_{j_{1}}=g^{*}\left(\alpha_{j}\right) \oplus \vartheta$ have different values and the random function $h$ generates two independent random variables so $y_{i_{2}}, y_{j_{2}}$ are independent from the input as well.
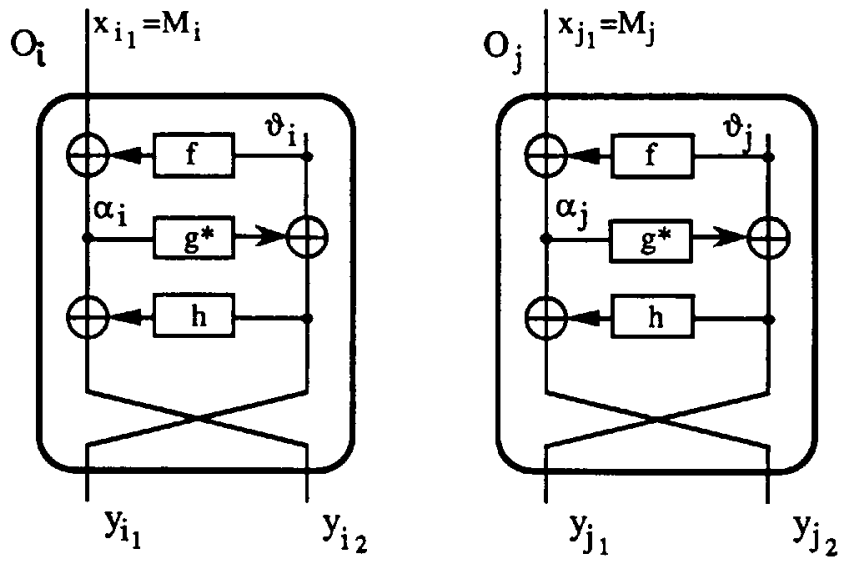

Figure 1: Two oracle gates evaluated using an $A$-code with $\psi\left(f, g^{*}, h\right)$

As this happens for all pairs of oracle gates, it means that all oracle gates generate independent random variables (from all inputs of oracle gates) when the oracle gates are evaluated by the randomized $A$-code $\mathcal{A}_{n}$.

Theorem 3.1 basically states that perfect security for messages of length $n$ can be achieved if each message is padded by a randomly chosen string of length at least $n$ and a single L-R randomizer $\psi\left(f, g^{*}, h\right)$ is used for diffusing the random information over the whole block. The random permutation $g^{*}$ can be substituted by identity permutation $(g=1)$ as $\vartheta_{i}$ and $\vartheta_{j}$ are already independent.

It is interesting to note that if the redundant strings $\vartheta_{i}(1 \leq i \leq r, r$ is the number of oracle gates) are kept constant for all messages (source states), the system will still be perfect if a second L-R randomizer is added. 
Let the random string $\vartheta$ be fixed (and publicly known - accessible to the distinguisher) for the whole transmission session i.e., $\vartheta_{i}=\vartheta$ for all $i=1, \ldots, r$. It means that the randomizer $\Psi_{2}=\psi_{1}(f, g, h) \circ \psi_{2}\left(h, g^{*}, f\right)$ is fed by the pair of random variables: the current message $M_{i}$ and $\vartheta$.

Theorem 3.2 Given $A$-code $\mathcal{A}_{n}=\left\{A^{n, 2 \pi}\right\}$. Assume that in the first stage, each message is assigned a common random variable $\vartheta \in I_{n}$ (the distinguisher knows it but it cannot change its value), then $\mathcal{A}_{n}$ is perfect provided the following two conditions are satisfied:

- the redundant string $\vartheta$ is placed to the left hand input of $L-R$ randomizer (Figure 2) i.e., $x_{i_{1}}=\vartheta$ and $x_{i_{2}}=M_{i}$ where $i=1, \ldots, r$ ( $r$ is the number of oracle gates in the distinguishing circuit; $r \leq 2^{n}$ ),

- the randomizer used in the second stage consists of cancatenation of two $L-R$ elementary randomizers i.e., $\Psi=\Psi_{2}=\psi_{1}(f, g, h) \circ \psi_{2}\left(h, g^{*}, f\right)$ (where $f, g, h \in_{R} F_{n}$, and a permutation $\left.g^{*} \in_{R} F_{n}\right)$.
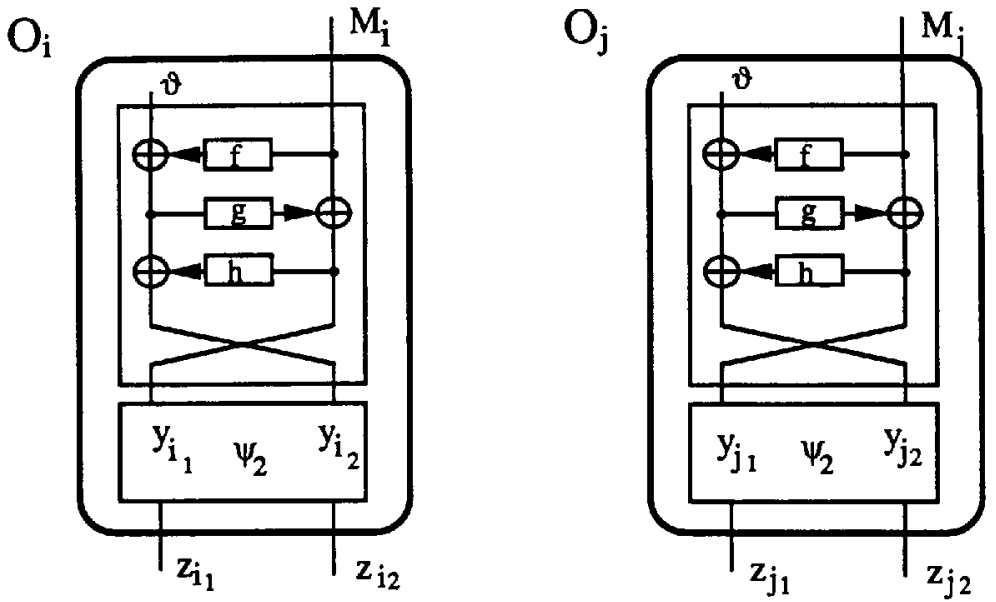

Figure 2: Oracle gates evaluated using an $A$-code with $\Psi_{2}$

Proof: As in the previous theorem, we consider a pair of oracle gates $\left(O_{i}, O_{j}\right)$ where $0 \leq$ $i<j \leq r$ and we prove that their outputs are independent from the inputs. Figure 2 shows the two oracle gates $\left(O_{i}, O_{j}\right)$. As the random variables $M_{i}, M_{j}$ have always different values, the random variables $y_{i_{2}}$ and $y_{j_{2}}$ are independent from the input (the random function $f$ generates independent raudom variables for different arguments). Now consider the second pair of the outputs $y_{i_{1}}$ and $y_{j_{1}}$. They are also independent as long as the random function $g$ generates two independent random variables (this happens only if the values of $\vartheta \oplus f\left(M_{i}\right)$ and $\vartheta \oplus f\left(M_{j}\right)$ are different). Otherwise, $y_{i_{1}}$ and $y_{j_{1}}$ are related and then

$$
g\left(\vartheta \oplus f\left(M_{i}\right)\right)=g\left(\vartheta \oplus f\left(M_{j}\right)\right)=Y
$$

This may happen if the random function $f$ collides i.e. $f\left(M_{i}\right)=f\left(M_{j}\right)$ (in [2] this case was called $X$ is $b a d)$. So we have

$$
y_{i_{1}}=M_{i} \oplus Y \text { and } y_{j_{1}}=M_{j} \oplus Y
$$


Note that although related, the random variables $y_{i_{1}}, y_{j_{1}}$ take on different values. Now if we put $\left(y_{i_{1}}, y_{i_{2}}\right)$ and $\left(y_{j_{1}}, y_{j_{2}}\right)$ to $\psi_{2}$ of $O_{i}$ and $O_{j}$, respectively, we face the same situation as in the previous theorem and the final result follows.
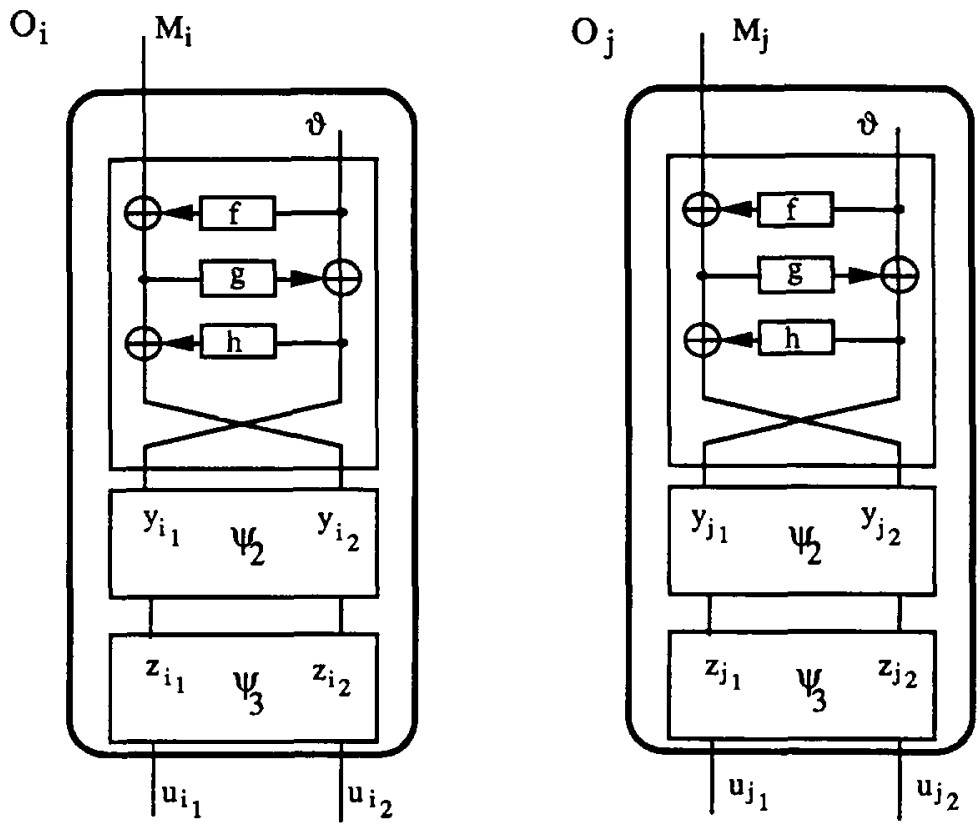

Figure 3: Oracle gates evaluated using an $A$-code with $\Psi_{3}$

Note that Theorem (3.2) is still true if we substitute the random function $h$ in $\psi_{1}$ by the constant 0 function. Hence the $A$-code $\mathcal{A}_{n}\left(\Psi_{2}\right)$, where $\Psi_{2}=\psi_{1}(f, g, \mathbf{0}) \circ \psi_{2}\left(f, g^{*}, h\right)$, is also perfect.

It is easy to check that, $\mathcal{A}_{n}\left(\Psi_{2}\right)$ is not perfect if the redundant string $\vartheta$ and the message $M_{i}$ are swopped i.e.,

$$
\begin{aligned}
& x_{i_{1}}=M_{i} \\
& x_{i_{2}}=\vartheta
\end{aligned}
$$

However, it will be perfect if we add another $\mathrm{L}-\mathrm{R}$ randomizer so the resulting $A$-code applies the randomizer $\Psi_{3}=b_{1}(f, g, h) \circ \psi_{2}\left(h, g^{*}, f\right) \circ \psi_{3}\left(h, g^{*}, f\right)$. Let us consider the resulting $A$-code. The random variable $v$ is fixed for all oracle gates so the function $f$ assigns the same random variable $f(\vartheta)$ for all gates. The random variables $M_{i} \oplus f(\vartheta)(i=1, \ldots, r)$ always take on different values for all oracle gates. So the random function $g$ assigns $r$ independent random variables and all outputs $y_{i_{1}}$ are independent from the inputs. Let us consider a single pair of oracle gates $\left(O_{i}, O_{j}\right)$ (Figure 3). We have already concluded that $y_{i_{1}}=g\left(M_{i} \oplus f(\vartheta)\right) \oplus \vartheta$ and $y_{j_{1}}=g\left(M_{j} \oplus f(\vartheta)\right) \oplus \vartheta$ are independent from $M_{i}$ and $M_{j}$. The second pair $y_{i_{2}}, y_{j_{2}}$ is also independent from the input if $g\left(M_{i} \oplus f(\vartheta)\right) \neq g\left(M_{j} \oplus f(\vartheta)\right)$. However if they collide, i.e. $Y=g\left(M_{i} \oplus f(\vartheta)\right)=g\left(M_{j} \oplus f(\vartheta)\right)$, the random variables $y_{i_{2}}=M_{i} \oplus f(\vartheta) \oplus h(Y)$ and $y_{j_{2}}=M_{j} \oplus f(\vartheta) \oplus h(Y)$ are related although they take on different values. 
Therefore if outputs $y_{i_{2}}, y_{j_{2}}$ are related to the input, they always have different values (and $\left.y_{i_{1}}=y_{j_{1}}\right)$. Note that $y_{i_{1}}, y_{j_{1}}$ are independent. The second randomizer $\psi_{2}$ does not change the situation - $z_{i_{2}}, z_{j_{2}}$ are independent from the input but $z_{i_{1}}, z_{j_{1}}$ may be related to the input (with some probability). According to Theorem (3.1), $\psi_{3}$ cuts the statistical relation with the input.

Note that if $x_{i_{1}}=\vartheta$ and $x_{i_{2}}=M_{i}$ (for $\left.i=1, \ldots, r\right)$, then the outputs $\left(z_{i_{1}}, z_{i_{2}}\right)$ are already independent from the input (see Theorem (3.2)) and $\psi_{3}$ preserves the independency.

Thus, we have shown that the following theorem is true.

Theorem 3.3 Given $A$-code $\mathcal{A}_{n}=\left\{A^{n, 2 n}\right\}$. Assume that in the first stage, each message is assigned a common random string $\vartheta \in I_{n}$ (the distinguisher knows it but it cannot change its value), then $\mathcal{A}_{n}$ is perfect provided the following two conditions are satisfied:

- the redundant string $\vartheta$ is placed to the same half of input of $L-R$ randomizer (Figure 9 ) i.e., $\left(x_{i_{1}}=M_{i}\right.$ and $\left.x_{i_{2}}=\vartheta\right)$ or $\left(x_{i_{1}}=\vartheta\right.$ and $\left.x_{i_{2}}=M_{i}\right)$, where $i=1, \ldots, r$ ( $r$ is the number of oracle gates in the distinguishing circuit; $r \leq 2^{n}$ ),

- the randomizer used in the second stage consists of concatenation of three $L-R$ elementary randomizers i.e., $\Psi=\Psi_{3}=\psi_{1}(f, g, h) \circ \psi_{2}\left(h, g^{*}, f\right) \circ \psi_{3}\left(h, g^{*}, f\right)$ (where $f, g, h \in_{R} F_{n}$ )

Note that the $A$-code considered in Theorem 3.3 is perfect even if the redundant string $\vartheta$ is made public (although public it cannot be changed by the distinguisher).

The above results can be summarised as follows:

$A$-codes $\mathcal{A}_{2}(\Psi)$ are perfect if:

- $\Psi=\psi_{1}\left(f, g^{*}, h\right)$ and the inputs to the randomizer are $x_{i_{1}}=M_{i}$ and $x_{i_{2}}=\vartheta_{i}$, where $\vartheta_{i}$ is independently selected for each message $M_{i}$ with the uniform probability distribution.

- $\Psi=\psi_{1}(f, g, h) \circ \psi_{2}\left(h, g^{*}, f\right)$ and the inputs to the randomizer are $x_{i_{1}}=\vartheta$ and $x_{i_{2}}=M_{i}$, where the random variable $\vartheta$ is fixed for the whole session (for all messages),

- $\Psi=\psi_{1}(f, g, h) \circ \psi_{2}\left(h, g^{*}, f\right) \circ \psi_{3}\left(h, g^{*}, f\right)$ and the inputs to the randomizer are either $\left(x_{i_{1}}=M_{i}\right.$ and $x_{i_{2}}=\vartheta$ ) or $\left(x_{i_{1}}=\vartheta\right.$ and $\left.x_{i_{2}}=M_{i}\right)$, where the random variable $\vartheta$ and its position in the input are fixed for the whole session (for all messages).

We have discussed the case of $A$-codes $\mathcal{A}_{n}(\Psi)$ when the length of redundant strings is the same as the length of messages. The same comments can be made for the case of $A$-codes when redundant strings are longer than messages.

Randomized $A$-codes are perfect if all outputs of the oracle gates (evaluated using the $A$ code) are independent from the input random variables. It also means that perfect $A$-codes resist the spoofing attack of order $T=r$ (where $r$ specifies the number of oracle gates in distinguishing circuits). Hence the knowledge of $r$ message/cryptogram pairs does not reveal any information to the attacker in forming a substitution message and their best strategy is random selection with uniform distribution from the rest of cryptograms $([3],[5])$. Therefore if an enemy wished to make the receiver accept a false cryptogram, they would have to select such a cryptogram randomly from the rest of $2^{m}-r$ elements. The probability of an enemy's success is equal to:

$$
\frac{2^{n}-r}{2^{m}-r}
$$

where $2^{n}$ is the size of the message space and $2^{m}$ is the size of the cryptogram space.

There is a problem with an implementation of randomized $A$-codes, as their random functions $f, g, h$ are not polynomially computable and to store them in a computer, requires exponential size of memory. To make the $A$-code implementable, the random functions $f, g, h \in_{R} F_{n}$ in L-R randomizers have to be substituted by pseudorandom ones $\left(f, g, h \in_{P S} F_{n}\right)$. It is easy to check that the resulting $A$-code is pseudorandom and its quality depends upon the quality of pseudorandom functions used. 


\section{Conclusions}

It is well known [6] that secrecy and authenticity are related. If the message source has redundancy, then any cryptosystem applied for secrecy also gives authentication of messages. If the message source has no redundancy, then any cryptogram injected by an enemy is accepted by the receiver as a genuine one. In general, to provide authenticity, it is necessary to introduce redundancy, which divides redundant messages into two classes. The first one consists of all meaningful messages whose cryptograms can appear in the communication channel. All meaningless redundant messages create the second class. Their cryptograms never occur in the channel (see [4]).

We defined randomized $A$-codes and gave some constructions for them. Our considerations are limited to the case when $n$-bit long messages are transformed into $2 n$-bit cryptograms. In these codes, the assignment of the cryptogram to a given message is done in two stages. The first introduces redundancy and the second applies the concatenation of $i$ Luby-Rackoff randomizers $(i=1,2,3)$.

The number of required stages in an L-R randomizer is directly affected by the way the redundancy is introduced. If a known constant padding is used for all messages (this padding sequence can be made public) but its position in the randomizer input (although fixed for the session) is not known, concatenation of three $L-R$ randomizers guarantees that the resulting $A$-code is perfect. Perfection implies that the authentication code is indistinguishable from a truly random function generator $F^{n, m}$ which assigns independently and uniformly distributed random cryptograms for each message (the number of oracle gates $r \leq 2^{n}$ ).

If two communicating parties agree beforehand that they will always place the redundant string at the left hand input of the randomizer, then it is possible to obtain a perfect $A$-code for a simpler randomizer $\Psi=\Psi_{2}$ that applies the concatenation of two L-R randomizers. Note that the positions of redundant string and message are important - the message should always be input to the right hand input of the randomizer (to the input of the random function $f$ ).

To be perfect, a randomized $A$-code with a single L-R randomizer needs to assign an independent and uniformely distributed random variable $\vartheta_{i}$ for each message $M_{i}$.

Perfect randomized $A$-codes have the advantage that they are resistant to a spoofing of order $T$ attack, as an enemy has to select a cryptogram from the remaining equally probable elements and their probability of success is strictly related to the redundancy.

Perfect randomized $A$-codes can be readily converted to pseudorandom $A$-codes whose quality depends on the quality of underlying pseudorandom functions used in L-R modules.

According to the definition, perfect $A$-codes do not leak information about the input to the output as all outputs are independent random variables. However, some information about the $A$-code structure can be extracted from the output.

\section{ACKNOWLEDGMENT}

We would like to thank Jacques Patarin, Babak Sadeghiyan and Yuliang Zheng for their critical comments and help. We thank Professor Jennifer Seberry for her continuous support and encouragement. Also we thank Cathy Newberry, Thomas Hardjono and other members of the CCSR for their assistance.

\section{References}

[1] O. Goldreich, S. Goldwasser, and S. Micali. How to construct random functions. Journal of the $A C M, 33(4): 792-807$, October 1986.

[2] M. Luby and Ch. Rackoff. How to construct pseudorandom permutations from pseudorandom functions. SIAM Journal on Computing, 17(2):373-386, April 1988. 
[3] J.L. Massey. Cryptography - a selective survey. In Digital Communication. Elsevier Science Publishers, 1986.

[4] R. Safavi-Naini and J. Seberry. Error correcting codes for authentication and subliminal channels. to appear in IEEE Transactions on Information Theory.

[5] R. Safavi-Naini and J. Seberry. On construction of practically perfect authentication codes. Technical report, Department of Computer Scienee, The University of New South Wales, ADFA, 1990.

[6] G.V. Simmons. A survey of information authentication. Proceedings of $I E E E, 76: 603-620$, 1988.

[7] Andrew C. Yao. Theory and application of trapdoor functions. In Proceedings of the 29rd IEEE Symposium on Fundation of Computer Science, pages 80-91, New York, 1982. IEEE.

[8] Y. Zheng, T. Matsumoto, and H. Imai. On the construction of block ciphers provably secure and not relying on any unproved hypotheses. Abstracts of CRYPTO'89, Santa Barbara, CA, July 1989. 